\title{
Biomarkers in endometrial cancer: Possible clinical applications (Review)
}

\author{
KOUJI BANNO, IORI KISU, MEGUMI YANOKURA, KOSUKE TSUJI, KENTA MASUDA, \\ ARISA UEKI, YUSUKE KOBAYASHI, WATARU YAMAGAMI, HIROYUKI NOMURA, \\ EIICHIRO TOMINAGA, NOBUYUKI SUSUMU and DAISUKE AOKI
}

Department of Obstetrics and Gynecology, School of Medicine, Keio University, Tokyo, Japan

Received February 10, 2012; Accepted March 12, 2012

DOI: $10.3892 / 01.2012 .654$

\begin{abstract}
The number of cases of endometrial cancer has shown a tendency to increase in recent years. Endometrial cancer originates from the endometrium and is classified, based on the development mechanism, into types 1 and 2, which are responsive and non-responsive to estrogen, respectively, and have significantly different gene expression profiles. Studies of genes with abnormal expression in endometrial cancer have identified multiple oncogenes, tumor suppressors, mismatch repair genes, apoptosis-associated genes, levels of hormone receptors and DNA ploidy and aneuploidy as biomarkers of endometrial cancer. The use of these molecules and genes may facilitate accurate diagnosis and prognostic prediction and contribute to individualized treatment. Trials of drugs which target these biomarkers and searches for new biomarkers using cDNA microarrays and RT-qPCR are ongoing and it is likely that these findings can be translated to clinical use.
\end{abstract}

\section{Contents}

1. Introduction

2. Differences in biomarkers in subtypes of endometrial cancer

3. Oncogenes

4. Tumor suppressors

5. Mismatch repair genes and microsatellite instability

6. Other biomarkers

7. Biomarkers for prognosis and treatment

8. Conclusion

Correspondence to: Dr Kouji Banno, Department of Obstetrics and Gynecology, School of Medicine, Keio University, Shinanomachi 35 Shinjuku-ku, Tokyo 160-8582, Japan

E-mail: kbanno@sc.itc.keio.ac.jp

Key words: endometrial cancer, biomarker, DNA microarray, prognosis, K-ras, PTEN

\section{Introduction}

Endometrial cancer is the seventh most common cancer worldwide in women (1). The incidence differs depending on the region: in developed countries, 10-20 in 100,000 women have endometrial cancer, whereas the incidence is approximately one-tenth this level in developing countries. The number of cases is rapidly increasing in Japan. Obesity, nulliparity and the administration of tamoxifen have been identified as risk factors for endometrial cancer (2).

In the last 10 years, numerous studies have aimed to identify tumor biomarkers. The US National Cancer Institute (NCI) defines a biomarker as 'a biological molecule found in blood, other body fluids, or tissues that is a sign of a normal or abnormal process, or of a condition or disease'. According to this definition, a biomarker includes not only the proteins normally used as tumor markers, such as CA-125, but also genes and chromosomes. Biomarkers of endometrial cancer that have been identified include the $K$-ras, HER2/neu, epithelial growth factor receptor $(E G F R)$, phosphatidylinositol 3-kinase catalytic subunit $(P I 3 K C A)$ and fibroblast growth factor receptor 2 (FGFR2) oncogenes; the phosphatase and tensin homolog $(P T E N), p 53, p 21$ and cyclin-dependent kinase inhibitor 2A (CDKN2A) cancer suppressor genes; the $h M L H 1, h M S H 2$, hMSH6, PMS1 and PMS2 mismatch repair genes; Ki-67, an index of cell proliferation; BCL2-associated X protein (Bax), an apoptosis promotor gene; $\mathrm{Bcl}-2$, an apoptosis suppressor; expression levels of estrogen and progesterone receptors; microvascular density (MVD); and vascular endothelial growth factor $\mathrm{A}(V E G F-A)$, which are all indices of angiogenesis; expression changes in $\mathrm{E} / \mathrm{P}$-cadherin and $\beta$-catenin, which are associated with infiltration and metastatic capacity; and ploidy and aneuploidy of DNA. The characteristics and functions of each of these biomarkers are described in this review.

\section{Differences in biomarkers in subtypes of endometrial cancer}

Endometrial cancer is classified into type 1 estrogen-responsive and type 2 estrogen-non-responsive cases, based on the mechanism of development. Type 1 endometrial cancer, caused by estrogen stimulation, is often observed in perimenopausal middle-aged women and has a well-differentiated histological 
type. By contrast, type 2 endometrial cancer is more frequent in older women following menopause, is moderately- to poorly-differentiated and has a poor prognosis. Engelsen et al examined the rates of mutation in each biomarker for endometrial cancer of the respective types (Table I) (3). The estrogen receptor (ER), progesterone receptor (PR), K-ras and PTEN are mutated at high rates in type 1 cases, whereas the levels of $p 53$ and HER2/neu mutation are high in type 2 cases, similar to the developmental mechanisms of the two types of endometrial cancer. However, the rate of mutation is not always associated with its significance, since a rare mutation may be important in carcinogenesis. The correlation between prognosis and biomarkers (Table II) reported by Engelsen et al should be viewed in the same way: biomarkers with high occurrence rates may not be strongly associated with prognosis and determining the significance of a biomarker using only epidemiological analysis is difficult.

\section{Oncogenes}

Oncogenes that have been reported as biomarkers of endometrial cancer include $K$-ras, HER2/neu, EGFR, PI3KCA and $F G F R 2$. These oncogenes are normally inactivated and their activation causes cell division (4). K-ras is associated with signal transduction in growth and differentiation and has a high rate of mutation in pancreatic and colorectal cancer. The mutation of $K$-ras has also been found in $10-30 \%$ of cases of endometrioid cancer (5-7). HER-2/neu is a member of the receptor tyrosine kinase (RTK) family, EGFR serves as a trigger for cell proliferation through the Ras-Raf-MAP kinase pathway and $P I 3 K$ and $A K T$ are downstream serine/threonine kinases (8). The overexpression of HER-2/neu occurs in 9-30\% of cases of endometrioid cancer and is frequently observed in non-endometrioid tumors $(9,10)$. HER-2/neu amplification in endometrial cancer is relatively rare and immunohistochemical data are inconsistent (11). The mutation of PI3KCA occurs in 24-36\% of cases of endometrioid cancer and a mutation in PTEN is simultaneously observed in 14-26\% of these cases (12). A study concerning the copy numbers and expression levels of these genes in endometrial cancer indicated that the amplification of $P I 3 K C A$ is associated with the activity of PI3K and suggested that PI3K is an influential target of anticancer drugs (13). In recent years, the mutation of FGFR2, another tyrosine kinase, has been found in $12 \%$ of cases of endometrial cancer. FGF is mitogenic for various cell types and is associated with regulation and tumor angiogenesis and metastasis (14). The mutation of FGFR2 causes carcinogenesis, which indicates that the inhibition of the activity of mutated FGFR2 may be a therapeutic approach and that FGFR2 is a potential candidate molecular target for the treatment of endometrial cancer (15).

\section{Tumor suppressors}

Tumor suppressor genes, which have been reported as biomarkers of endometrial cancer, include PTEN, p53, p21 and CDKN2A/ p16. PTEN encodes a phosphatase that antagonizes the PI3K/ AKT pathway and a reduction in PTEN activity increases cell proliferation and induction of angiogenesis and changes cell adhesion and migratory capacity (16). PTEN may be inactivated by mutation, deletion or promoter hypermethylation (17). PTEN is the most frequently mutated gene in endometrial cancer, with a rate of $25-83 \%$ among all cases and a particularly high rate in endometrioid adenocarcinoma and tumors with microsatellite instability (MSI) (18). The mutation of PTEN is also observed in endometrial hyperplasia, suggesting that PTEN mutation is an early event in carcinogenesis (19). Activation of the P13K/ AKT and mammalian target of rapamycin (mTOR) signaling pathways occurs concomitantly with the loss of PTEN function in most cases of endometrioid adenocarcinoma, suggesting that the carcinogenic mechanism involves mTOR inhibition (20). p53 is a common cancer suppressor gene that governs the cell cycle, apoptosis and differentiation (21). When DNA is damaged, p53 serves as a brake to stop the cell cycle by enhancing the transcription of $p 21$, an inhibitor of cyclin-dependent kinase. Normal p53 undergoes rapid degradation, but non-functional mutated p53 is resistant to degradation and the mutation and overexpression of p53 are simultaneously reported in $76 \%$ of cases with a p53 mutation (22). In endometrial cancer, the overexpression of p53 occurs in $15-30 \%$ of cases and the mutation of $p 53$ is found in $10-20 \%$ of cases (23-26). It has been proposed that the overexpression of p53 is observed more frequently in serous adenocarcinoma than in endometrioid cancer, with the mutation of p53 occurring in the early development of serous adenocarcinoma and in the later development of endometrioid adenocarcinoma (27). The inactivation of $C D K N 2 A / p 16$, a cell cycle regulation factor, is observed in a number of malignant tumors and is more frequently caused by the loss of protein expression, homozygous deletion and promoter methylation, and less frequently by mutation (28). In endometrial cancer, the loss of expression of p16 is observed in $14-74 \%$ of cases; however, mutation, deletion and promoter methylation are recognized in only 2-6\% of cases (29-31) and there are numerous unclear aspects of the inactivation mechanism of p16.

\section{Mismatch repair genes and microsatellite instability}

DNA repair and mismatch repair systems are important in maintaining genetic stability. Simple genomic repeat sequences are particularly vulnerable to replication errors. This phenomenon is referred to as MSI and is thought to result from the accumulation of mutations during DNA replication and to be associated with mismatch repair (MMR) gene mutation (32). MSI was initially identified as a result of the mutation of MMR-associated genes, including $h M L H 1, h M S H 2, h M S H 6$, PMS1 and PMS2 in germ cell lines of patients with hereditary non-polyposis colorectal cancer (HNPCC) (33). MSI occurs in $20-30 \%$ of cases of endometrial cancer, with a major cause being epigenetic inactivation due to the hypermethylation of the $h M L H 1$ promoter $(34,35)$. MSI has also been reported to be more common in endometrioid cancer than in non-endometrioid cancer (36). Arvanitis et al suggested that the allelic imbalance in $h M L H 1$ was associated with the response of endometrial cancer to radiotherapy (37). Moreover, the MSI analysis prior to and following radiation treatment may be used as a marker of the clinical outcome of patients (37).

\section{Other biomarkers}

In addition to the oncogenes and cancer suppressor genes described in the previous sections, several other molecules 
Table I. Expression of biomarkers in type 1 and type 2 endometrial cancer.

\begin{tabular}{|c|c|c|c|c|}
\hline Target & Function & Change & Type $1(\%)$ & Type $2(\%)$ \\
\hline$K$-ras & Oncogene & Mutation & $13-26$ & $0-10$ \\
\hline HER-2/neu & Oncogene & Enhanced expression & Rare & $18-80$ \\
\hline$P I K 3 C A$ & Oncogene & Mutation & $26-36$ & $26-36$ \\
\hline FGFR2 & Oncogene & Mutation & 12 & 12 \\
\hline PTEN & Tumor suppressor & Mutation, deletion, methylation & $35-55$ & $0-11$ \\
\hline p53 & Tumor suppressor & Mutation & $5-10$ & $80-90$ \\
\hline pl6 & Cancer suppressor & Mutation, methylation, enhanced expression & 10 & $10-40$ \\
\hline$M L H 1$ & DNA repair & Methylation & $20-35$ & $0-10$ \\
\hline$B c l-2$ & Tumor suppressor & Mutation & 65 & 67 \\
\hline $\operatorname{Bax}$ & Oncogene & Mutation & 48 & 43 \\
\hline ER, PR & Transcription factor & Enhanced expression & $70-73$ & $19-24$ \\
\hline$\beta$-catenin & Oncogene & Mutation & $25-38$ & $0-5$ \\
\hline E-cadherin & Tumor suppressor & Mutation, methylation & $22-43$ & $57-75$ \\
\hline$E Z H 2$ & Transcription factor & Enhanced expression & 16 & 36 \\
\hline$B M I-1$ & Transcription factor & Enhanced expression & 53 & 62 \\
\hline
\end{tabular}

Table from ref. 3. PIK3CA, phosphatidylinositol 3-kinase catalytic subunit; FGFR2, fibroblast growth factor receptor 2; PTEN, phosphatase and tensin homolog; Bax, BCL2-associated X protein; ER, estrogen receptor; PR, progesterone receptor.

Table II. Biomarkers as prognostic predictors in endometrial cancer.

\begin{tabular}{ll}
\hline Evidence level & \multicolumn{1}{c}{ Biomarker } \\
\hline Consistent results obtained in & DNA ploidy \\
retrospective studies & $E R / P R$ \\
& $p 53$ \\
& $K i-67$ \\
& $B c l-2$ \\
Inconsistent results obtained in & $H E R$-2/neu \\
several studies & $P T E N$ \\
& $p 16$ \\
& MSI \\
& $\beta$-catenin \\
& $K$-ras \\
& Angiogenesis markers \\
An association with prognosis & (MVD, $V E G F$ - $A$, \\
suggested in a few studies & $V P I, V M I, G M P$ ) \\
& E-cadherin \\
& $P I 3 K$ signal activation
\end{tabular}

Table from ref. 3. PTEN, phosphatase and tensin homolog; ER, estrogen receptor; PR, progesterone receptor; $V E G F-A$, vascular endothelial growth factor A.

and genes have been identified as biomarkers of endometrial cancer. These include Ki-67, Bax, Bcl-2, the levels of ERs and PRs and DNA ploidy and aneuploidy. Cell proliferation may be quantified by counting the number of mitoses or by the immunohistochemical examination of Ki-67/MIB-1 expression. The expression of $\mathrm{Ki}-67$ is increased in serous adenoma, high-grade cancers and in invasive regions in endometrial cancer (38). Cancers also often involve loss of the regulation of apoptosis. In endometrial cancer, the expression of Bax, an apoptosis-promoting gene, may be lost due to mutation (39), while $\mathrm{Bcl}-2$, an anti-apoptosis factor, has an enhanced expression in endometrial hyperplasia and a reduced expression in endometrial cancer (40).

ERs and PRs present in the endometrium facilitate the estrogen-induced proliferation and differentiation of endometrial cells and the antagonism of these functions by progesterone, respectively (41). The selection of treatment may be made based on the levels of the hormone receptors and this approach has improved the treatment of endometrial and breast cancer. Differences in hormone-targeted therapies in types 1 and 2 endometrial cancer due to different ER signaling pathways are also of interest $(42,43)$. Progestogens act through the PR pathway and via other steroid receptors. The PR is required for the inhibition of endometrial proliferation caused by estrogen and downregulates the activities of estrogen by preventing the transactivation of $\mathrm{ER} \alpha$.

Chromosomal instability leading to structural and numerical abnormalities of chromosomes has a primary role in the malignant alteration of tumors. Chromosomal aneuploidy is found in 20-35\% of cases of endometrial cancer and is associated with advanced cancers, high-grade non-endometrioid cancer and deep myometrial invasion in the FIGO staging of carcinoma $(44,45)$.

Trials to identify new biomarkers using cDNA microarrays and RT-qPCR for the comparison of gene expression levels in normal and cancer tissues have been conducted. Using a cDNA microarray, Risinger et al identified 24 transcripts with significantly different expression levels in endometrial cancer tissues compared with those in normal tissues. Among 
these genes, five showed reduced expression levels in all specimens: paternally expressed 3 (PEG3); signal transducer and activator of transcription 12 (STAT12); REV3-like, catalytic subunit of DNA polymerase $\zeta(R E V 3 L)$; forkhead box O1 (FOXO1A); and forkhead box O4 (MLLT7) (46). Using a similar cDNA microarray analysis, Planagumà et al identified 53 genes with different expression levels in cancer and normal tissues. Two genes with particularly enhanced expression in cancer tissues were RUNX1/AML1, a proto-oncogene in acute myeloid leukemia and ETV5, an oncogene coding for the Ets transcription factor (47). These two genes also showed significant differences in expression by RT-qPCR and quantitative immunohistochemical analysis. Colas et al used gene expression analysis with RT-qPCR following the suction of endometrial cells from patients with endometrial cancer and identified 20 genes with significantly different expression levels compared with those in the normal endometria, including acetyl-CoA acyltransferase 1 (ACAA1), adaptor-related protein complex 1, mu-2 subunit (AP1M2), cingulin (CGN), discoidin domain receptor tyrosine kinase 1 (DDR1), EPS8like 2 (EPS8L2) and FAST kinase domain 1 (FASTKD1) (48). These include a gene associated with $\beta$ oxidation (ACAAI) and a gene of unknown function (EPS8L2) and the association with carcinogenesis is unclear. These findings require further examination using mutation analysis in tissue samples and the functional analysis of the genes themselves.

\section{Biomarkers for prognosis and treatment}

Many retrospective studies have examined the effects of molecular markers on prognosis (Table II) (3). Several studies have shown an association of aneuploidy with poor prognosis $(49,50)$, while the expression of steroid receptors is associated with higher survival rates and better treatment response (51). Among oncogenes, the overexpression of HER-2 is associated with a reduced survival rate, but the clinical meaning and the predictability of treatment response are not fully understood (52). The overexpression of p53 has also been associated with a poor prognosis by a plurality of retrospective studies and the loss of expression of p16 is similarly linked to a poor prognosis (53). The effect of the mutation of PTEN remains controversial, since this has been found to be positively associated with prognosis, whereas the loss of expression and methylation of PTEN are negatively associated with prognosis (54). A high proliferative activity of tumor cells based on Ki-67 expression and the number of mitoses is associated with a poor prognosis in endometrial cancer; however, the multivariate survival analyses do not show concordant results, which may be due to technical differences in measuring $\mathrm{Ki}-67(55,56)$. The loss of expression of $\mathrm{Bcl}-2$ and the resultant reduced apoptosis are also associated with a poor prognosis (39).

Drugs for molecularly targeted treatment are being explored for endometrial cancer. The biomarkers described in the previous sections are not necessarily the targets, but a number of biomarkers are under study as potential therapeutic targets, including aromatase, hormone receptors, EGFR tyrosine kinases, the VGFR family, PTEN as a downstream molecule in the $P I 3 K$ pathway and mTOR. Abnormalities in the $P I 3 K$ pathway are common in endometrial cancer and the use of analogs of wortmannin, a PI3K inhibitor, as drugs is being examined. A number of mTOR inhibitors, including temsirolimus, are being tested in phase 2 trials in the US (3). The efficacy and safety of cetuximab, gefitinib, erlotinib, lapatinib and trastuzumab (EGFR inhibitors) and aflibercept and bevacizumab (VGFR inhibitors) are also being examined in phase 2 trials.

\section{Conclusion}

The generalization and individualization of treatment are significant factors in cancer therapy. Evidence obtained from certain clinical trials cannot be applied to all patients and generalization and individualization of information is also needed in future biomarker studies. Biomarkers, including tumor markers such as CA19-9 and neuron-specific enolase (NSE), are currently used clinically as diagnostic clues. However, advances in molecularly targeted therapy based on tumor markers may facilitate the selection of treatment suitable for each patient through the targeting of various tyrosine kinaseassociated molecules and hormone receptors. Thus, future studies are likely to focus on biomarkers for endometrial cancer to identify new therapeutic targets and on the optimization of therapy based on the expression levels of these biomarkers.

Biomarkers such as $p 53$ and $B c l-2$ have become indices for the individualization of treatment and life-changing indices for patients as potential factors for the prediction of prognosis. In breast cancer, the Oncotype DX ${ }^{\circledR}$ tool, used to evaluate the risk of relapse based on the expression levels of a plurality of genes, has already been commercialized. In endometrial cancer, a convenient and accurate method for predicting prognosis and the risk of relapse for use in clinical practice may greatly contribute to areas such as the application of fertility-conserving treatment. However, a number of problems remain to be solved before this becomes a reality. At present, each biomarker provides only limited information and searching for biomarkers with higher sensitivity and specificity and the further refinement of test items are required for practical use. In addition, the quantification of gene expression by RT-qPCR often requires the suction of cells from the endometrium and improved methods are required for the evaluation of epigenetic changes such as those involving microRNA and sugar chain modification.

\section{Acknowledgements}

The authors gratefully acknowledge grant support from the Japan Society for the Promotion of Science (JSPS) through a Grant-in-Aid for Scientific Research (KAKENHI), a Grant-in-Aid for Scientific Research (C) (22591866) and a Grant-in-Aid for Young Scientists (B) (21791573); the Ichiro Kanehara Foundation; and the Keio University Medical Science Fund through a Research Grant for Life Sciences and Medicine.

\section{References}

1. World Health Organization, International Agency for Research on Cancer: World Cancer Report. IARC Press, Lyon, 2003.

2. Amant F, Moerman P, Neven P, Timmerman D, Van Limbergen E and Vergote I: Endometrial cancer. Lancet 366: 491-505, 2005.

3. Engelsen IB, Akslen LA and Salvesen HB: Biologic markers in endometrial cancer treatment. APMIS 117: 693-707, 2009. 
4. Vogelstein B and Kinzler KW: Cancer genes and the pathways they control. Nat Med 10: 789-799, 2004.

5. Enomoto T, Inoue M, Perantoni AO, Terakawa N, Tanizawa O and Rice JM: K-ras activation in neoplasms of the human female reproductive tract. Cancer Res 50: 6139-6145, 1990.

6. Caduff RF, Johnston CM and Frank TS: Mutations of the Ki-ras oncogene in carcinoma of the endometrium. Am J Pathol 146: $182-188,1995$

7. Mammas IN, Zafiropoulos A and Spandidos DA: Involvement of the ras genes in female genital tract. Int J Oncol 26: 1241-1255, 2005.

8. Cully M, You H, Levine AJ and Mak TW: Beyond PTEN mutations: the PI3K pathway as an integrator of multiple inputs during tumorigenesis. Nat Rev Cancer 6: 184-192, 2006.

9. Slomovitz BM, Broaddus RR, Burke TW, Sneige N, Soliman PT and Wu W: Her-2/neu overexpression and amplification in uterine papillary serous carcinoma. J Clin Oncol 22: 3126-3132, 2004.

10. Berchuck A, Rodriguez G, Kinney RB, Soper JT, Dodge RK and Clarke-Pearson DL: Overexpression of HER-2/neu in endometrial cancer is associated with advanced stage disease. Am J Obstet Gynecol 164: 15-21, 1991.

11. Morrison C, Zanagnolo V, Ramirez N, Cohn DE, Kelbick N and Copeland L: HER-2 is an independent prognostic factor in endometrial cancer: association with outcome in a large cohor of surgically staged patients. J Clin Oncol 24: 2376-2385, 2006.

12. Oda K, Stokoe D, Taketani Y and McCormick F: High frequency of coexistent mutations of PIK3CA and PTEN genes in endometrial carcinoma. Cancer Res 65: 10669-10673, 2005.

13. Salvesen HB, Carter SL, Mannelqvist M, Dutt A, Stefansson IM and Getz G: Integrated genomic profiling of endometrial carcinoma associates aggressive tumors with indicators of PI3 kinase activation. Proc Natl Acad Sci USA 106: 4834-4839, 2009

14. Soufla G, Sifakis S and Spandidos DA: FGF2 transcript levels are positively correlated with EFG and IGF-1 in the malignant endometrium. Cancer Lett 259: 146-155, 2008.

15. Dutt A, Salvesen HB, Chen TH, Ramos AH, Onofrio RC and Hatton C: Drug-sensitive FGFR2 mutations in endometrial carcinoma. Proc Natl Acad Sci USA 105: 8713-8717, 2008.

16. Tamura M, Gu J, Matsumoto K, Aota S, Parsons R and Yamada KM: Inhibition of cell migration, spreading, and focal adhesions by tumor suppressor PTEN. Science 280: 614-617, 1998.

17. Salvesen HB, MacDonald N, Ryan A, Jacobs IJ, Lynch ED and Akslen LA: PTEN methylation is associated with advanced stage and microsatellite instability in endometrial carcinoma. Int $J$ Cancer 91: 22-26, 2001

18. Salvesen HB, Stefansson I, Kretzschmar EI, Gruber P, MacDonald ND and Ryan A: Significance of PTEN alterations in endometrial carcinoma: a population-based study of mutations, promoter methylation and PTEN protein expression. Int J Oncol 25: 1615-1623, 2004.

19. Maxwell GL, Risinger JI, Gumbs C, Shaw H, Bentley RC and Barrett JC: Mutation of the PTEN tumor suppressor gene in endometrial hyperplasias. Cancer Res 58: 2500-2503, 1998

20. Prat J, Gallardo A, Cuatrecasas M and Catasus L: Endometrial carcinoma: pathology and genetics. Pathology 39: 72-87, 2007.

21. Vogelstein B and Kinzler KW: p53 function and dysfunction. Cell 70: 523-526, 1992

22. Soong R, Robbins PD, Dix BR, Grieu F, Lim B and Knowles S Concordance between p53 protein overexpression and gene mutation in a large series of common human carcinomas. Hum Pathol 27: 1050-1055, 1996.

23. Risinger JI, Dent GA, Ignar-Trowbridge D, McLachlan JA, Tsao MS and Senterman M: p53 gene mutations in human endometrial carcinoma. Mol Carcinog 5: 250-253, 1992.

24. Kihana T, Hamada K, Inoue Y, Yano N, Iketani H and Murao S Mutation and allelic loss of the p53 gene in endometrial carcinoma. Cancer 76: 72-78, 1995.

25. Soong R, Knowles S, Williams KE, Hammond IG, Wysocki SJ and Iacopetta BJ: Overexpression of p53 protein is an independent prognostic indicator in human endometrial carcinoma. Br J Cancer 74: 562-567, 1996.

26. Pisani AL, Barbuto DA, Chen D, Ramos L, Lagasse LD and Karlan BY: HER-2/neu, p53, and DNA analyses as prognosticators for survival in endometrial carcinoma. Obstet Gynecol 85 : 729-734, 1995

27. Lax SF, Kendall B, Tashiro H, Slebos RJ and Hedrick L: The frequency of p53, K-ras mutations, and microsatellite instability differs in uterine endometrioid and serous carcinoma: evidence of distinct molecular genetic pathways. Cancer 88: 814-824, 2000
28. Nuovo GJ, Plaia TW, Belinsky SA, Baylin SB and Herman JG: In situ detection of the hypermethylation-induced inactivation of the p16 gene as an early event in oncogenesis. Proc Natl Acad Sci USA 96: 12754-12759, 1999.

29. Nakashima R, Fujita M, Enomoto T, Haba T, Yoshino K and Wada H: Alteration of p16 and p15 genes in human uterine tumours. Br J Cancer 80: 458-467, 1999.

30. Salvesen HB, Das S and Akslen LA: Loss of nuclear p16 protein expression is not associated with promoter methylation but defines a subgroup of aggressive endometrial carcinomas with poor prognosis. Clin Cancer Res 6: 153-159, 2000.

31. Milde-Langosch K, Riethdorf L, Bamberger AM and Löning T: P16/MTS1 and pRB expression in endometrial carcinomas. Virchows Arch 434: 23-28, 1999.

32. Ionov Y, Peinado MA, Malkhosyan S, Shibata D and Perucho M Ubiquitous somatic mutations in simple repeated sequences reveal a new mechanism for colonic carcinogenesis. Nature 363: 558-561, 1993.

33. Lynch HT, Smyrk T and Lynch JF: Overview of natural history, pathology, molecular genetics and management of HNPCC (Lynch Syndrome). Int J Cancer 69: 38-43, 1996.

34. Caduff RF, Johnston CM, Svoboda-Newman SM, Poy EL, Merajver SD and Frank TS: Clinical and pathological significance of microsatellite instability in sporadic endometrial carcinoma. Am J Pathol 148: 1671-1678, 1996.

35. Esteller M, Levine R, Baylin SB, Ellenson LH and Herman JG: MLH1 promoter hypermethylation is associated with the microsatellite instability phenotype in sporadic endometrial carcinomas. Oncogene 17: 2413-2417, 1998.

36. Catasus L, Machin P, Matias-Guiu X and Prat J: Microsatellite instability in endometrial carcinomas: clinicopathologic correlations in a series of 42 cases. Hum Pathol 29: 1160-1164, 1998.

37. Arvanitis DA, Angelakis E, Koumantakis EE and Spandidos DA: Allelic imbalance in hMLH1 or BRCA2 loci associated with response of cervical and endometrial cancer to radiotherapy. Int J Mol Med 10: 55-63, 2002

38. Horree N, Van Diest PJ, Sie-Go DM and Heintz AP: The invasive front in endometrial carcinoma: higher proliferation and associated derailment of cell cycle regulators. Hum Pathol 38: 1232-1238, 2007.

39. Sakuragi N, Salah-eldin AE, Watari H, Itoh T, Inoue $\mathrm{S}$ and Moriuchi T: Bax, Bcl-2, and p53 expression in endometrial cancer. Gynecol Oncol 86: 288-296, 2002.

40. Ohkouchi T, Sakuragi N, Watari H, Nomura E, Todo Y and Yamada H: Prognostic significance of Bcl-2, p53 overexpression, and lymph node metastasis in surgically staged endometrial carcinoma. Am J Obstet Gynecol 187: 353-359, 2002.

41. Ito K, Utsunomiya $\mathrm{H}$, Yaegashi $\mathrm{N}$ and Sasano H: Biological roles of estrogen and progesterone in human endometrial carcinoma new developments in potential endocrine therapy for endometrial cancer. Endocr J 54: 667-679, 2007.

42. McDonnell DP and Norris JD: Connections and regulation of the human estrogen receptor. Science 296: 1642-1644, 2002

43. Shang Y: Molecular mechanisms of oestrogen and SERMs in endometrial carcinogenesis. Nat Rev Cancer 6: 360-368, 2006.

44. van der Putten HW, Baak JP, Koenders TJ, Kurver PH, Stolk HG and Stolte LA: Prognostic value of quantitative pathologic features and DNA content in individual patients with stage I endometrial adenocarcinoma. Cancer 63: 1378-1387, 1989.

45. Susini T, Amunni G, Molino C, Carriero C, Rapi S and Branconi F: Ten-year results of a prospective study on the prognostic role of ploidy in endometrial carcinoma: DNA aneuploidy identifies high-risk cases among the so-called 'low-risk' patients with well and moderately differentiated tumors. Cancer 109: 882-890, 2007

46. Risinger JI, Maxwell GL, Chandramouli GV, et al: Microarray analysis reveals distinct gene expression profiles among different histologic subtypes of endometrial cancer. Cancer Res 63: 72-87, 2003.

47. Planagumà J, Díaz-Fuertes M, Gil-Moreno A, et al: A differential gene expression profile reveals overexpression of RUNX1/AML1 in invasive endometrioid carcinoma. Cancer Res 64: 8846-8853, 2004.

48. Colas E, Perez C, Cabrera S, et al: Molecular markers of endometrial carcinoma detected in uterine aspirates. Int J Cancer 129: 2435-2444, 2011

49. Prat J: Prognostic parameters of endometrial carcinoma. Hum Pathol 35: 649-662,2004. 
50. Yamazawa K, Seki K, Matsui H, Kihara M and Sekiya S: Prognostic factors in young women with endometrial carcinoma: a report of 20 cases and review of literature. Int J Gynecol Cancer 10: 212-222, 2000

51. Singh M, Zaino RJ, Filiaci VJ and Leslie KK: Relationship of estrogen and progesterone receptors to clinical outcome in metastatic endometrial carcinoma: a Gynecologic Oncology Group Study. Gynecol Oncol 106: 325-333, 2007.

52. Slomovitz BM, Broaddus RR, Burke TW, Sneige N, Soliman PT and $\mathrm{Wu} \mathrm{W}$ : Her-2/neu overexpression and amplification in uterine papillary serous carcinoma. J Clin Oncol 22: 3126-3132, 2004.

53. Salvesen HB, Iversen OE and Akslen LA: Prognostic significance of angiogenesis and Ki-67, p53 and p21 expression: a populationbased endometrial carcinoma study. J Clin Oncol 17: 1382-1390, 1999.
54. Salvesen HB, Stefansson I, Kalvenes MB, Das S and Akslen LA: Loss of PTEN expression is associated with metastatic disease in patients with endometrial carcinoma. Cancer 94: 2185-2191, 2002.

55. Nordström B, Strang P, Bergström R, Nilsson S and Tribukait B: A comparison of proliferation markers and their prognostic value for women with endometrial carcinoma. Cancer 78: 1942-1951, 1996.

56. Al Kushi A, Lim P, Quino-Parsons C and Gilks CB: Markers of proliferative activity are predictors of patient outcome for low-grade endometrioid adenocarcinoma but not papillary serous carcinoma of endometrium. Mod Pathol 15: 365-371, 2002. 